\title{
GLOBALIZACIÓN Y DESEMPLEO. REFLEXIONES EMPÍRICAS DESDE LA PERSPECTIVA DE LA TEORÍA DEL "SISTEMA MUNDIAL"
}

\section{GLOBALIZATION AND UNEMPLOYMENT. EMPIRICAL REFLECTIONS FROM THE WORLD-SYSTEM THEORY PERSPECTIVE}

\section{Arno Tausch*}

\author{
RESUMEN
}

Este artículo demuestra la relevancia de las teorías cuantitativas de la dependencia $y$ del sistema mundial en los intentos de explicar el desempleo tanto al nivel del sistema mundial como al nivel de las regiones Europeas. Se demuestran que existen correlaciones negativas a largo plazo entre la penetración alta del capital extranjero (concepto UNCTAD) y el empleo a nivel mundial; esta correlación se mantiene también en un estudio del desarrollo regional en la Unión Europea, lo que apunta a plantear una revisión de la estrategia propuesta por la Comisión Europea para desarrollar una respuesta a nivel de la Unión Europea en materia de política industrial.

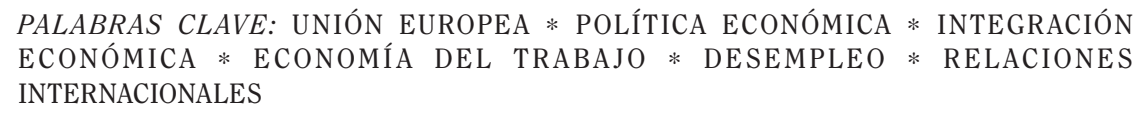

\section{ABSTRACT}

This article shows the relevance of quantitative theories of dependency and the world system for the explanation of unemployment both on the global system level and on the level of European regions. There are long-term negative correlations between a high penetration of foreign capital (UNCTAD concept) and employment performance at the world level, and this negative effect of a high penetration by foreign capital is also observed in a study of regional development in the European Union. This led to the conclusion that there is need for changing the policy proposed by the European Commission for developing a EU strategy with an industrial policy.

KEYWORDS: EUROPEAN UNION * ECONOMICS POLICY * ECONOMIC INTEGRATION * LABOUR ECONOMICS $*$ UNEMPLOYMENT $*$ INTERNATIONAL RELATIONS

Profesor Visitante (Visiting Professor) de Ciencias Económicas, Universidad de Corvinus (Corvinus University), Budapest $y$ en el Departamento de
Ciencias Políticas de la Universidad de Innsbruck, Austria.

arno.tausch@yahoo.de 


\section{INTRODUCCIÓN ${ }^{1}$}

¿Es la globalización una garantía para el éxito en el desarrollo, sobre todo el empleo, especialmente en los nuevos Estados miembros de la Unión Europea? O conviene sacar una lección ofrecida por la experiencia de la periferia y semi-periferia del mundo, y ¿debe Europa, al final de la cuenta comenzar a estudiar las experiencias de América Latina $y$ las Ciencias Sociales globales que subrayaron más que 300 estudios, publicadas en las principales revistas de Sociología y de Ciencias Políticas - sobre todo en Estados Unidos- que la dependencia en general tiene efectos críticos a largo plazo y lleva a la polarización de las relaciones sociales, tal como se puede observar hoy en día en Europa? Esta perspectiva radical significa una ruptura fundamental con el pensamiento dominante existente sobre el tema (véase, por ejemplo Eco-efficient Growth in the Age of Globalisation. Swedish non-paper on the Lisbon strategy post-2010, Stockholm, 2009).

Aquellos quienes comparten la crítica radical de la globalización se hacen más numerosos en los últimos años, tanto al nivel académico como al nivel político. Muy tem-

1 Original: Castellano. El autor agradece al Instituto Cervantes de Viena $y$ al profesor Christian Ghymers Brusellas por la oportunidad de correguir el texto en el idioma Castellano. prano, el científico social noruego Johan Galtung (1982) retrataba ya la Unión Europea como nada más que una poderosa máquina de intervenciones al servicio de las empresas transnacionales. En la recientes elecciones al Parlamento Europeo en 2009, los partidos políticos que critican la posición de la antigua Comisión y que no pertenecen a las principales agrupaciones de centro-derecha o centroizquierda en el Parlamento Europeo, PPE-DE (Partido Popular Europeo), PES (socialdemócratas), ALDE (liberales), EFA (verdes), ganaron más del $30 \%$ del voto popular en 13 de los 27 miembros de la UE. Críticos de la globalización, como la plataforma "Attac", desempeñan un papel cada vez más importante en la política de varios países de la Unión Europea y contribuyeron también a la derrota del gobierno francés en el referéndum sobre la Constitución Europea en 2005.

Tanto las tasas de globalización como las tasas de desempleo en Europa se parecen mucho más a las tasas correspondientes latinoamericanas y no europeas (ver nuestras propias compilaciones con base en United Nations Statistics <http://unstats.un.org/unsd/ Demographic/Products/socind/unemployment. htm>). El mapa del desempleo global muestra muy claramente que el desempleo es mucho más fuerte en los países de Europa y en la semiperiferia en general, que en los Estados Unidos y las otras economías ricas de las democracias occidentales del OCDE: 
MAPA 1

DESEMPLEO EN EL SISTEMA MUNDIAL

\section{tasas de desempleo}

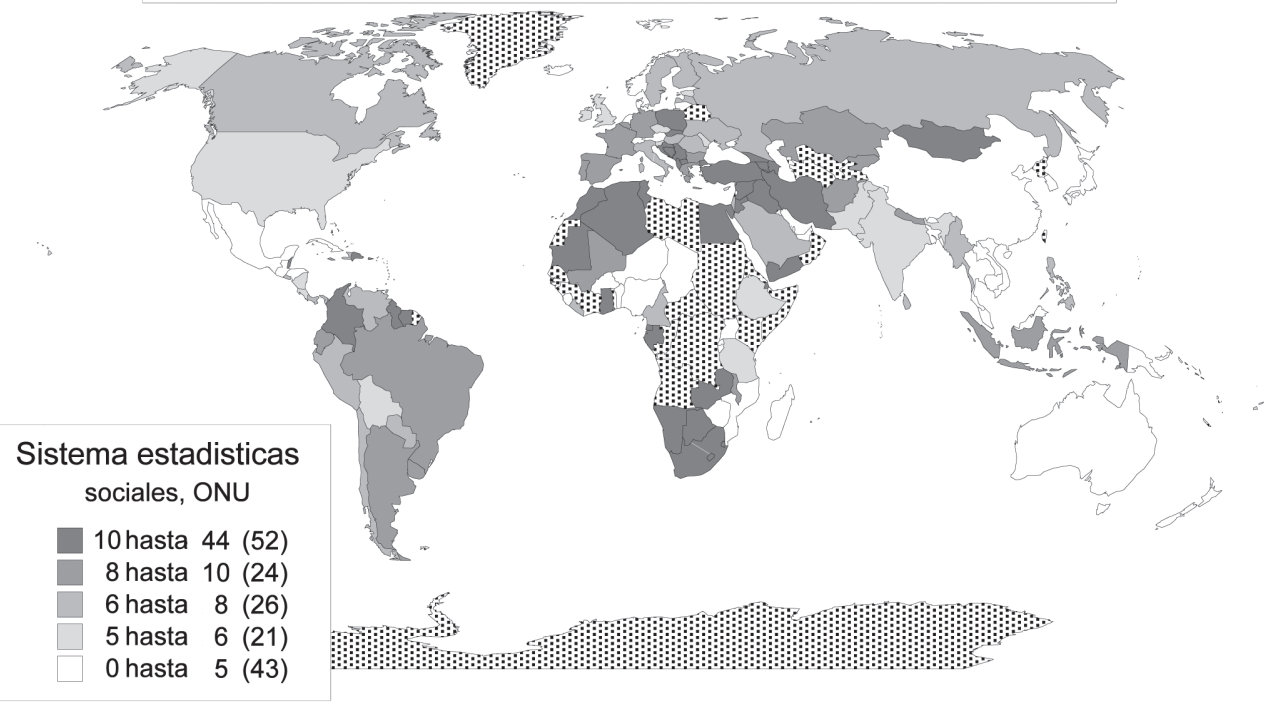

Fuente: nuestras propias compilaciones con base en United Nations Statistics <http://unstats.un.org/unsd/Demographic/ Products/socind/unemployment.htm> Color oscuro: alto desempleo. No hay datos disponibles para Groenlandia, Bielorrusia, varios países Africanos y en Asia Central.

\section{UN ANÁLISIS CRITICO DE CIERTOS EFECTOS DE LA PENETRACIÓN POR EL CAPITAL TRANSNACIONAL}

Ya fueron presentadas recientemente -en la Revista del Ministerio de Trabajo e Inmigración del correspondiente Ministerio del país de turno de la presidencia de la Unión Europea en el primer semestre 2010, Españalas conclusiones de las Ciencias Sociales latinoamericanas desde los años 1970 para explicar el fracaso del proceso de Lisboa en Europa (Tausch y Galindo-Lucas, 2009). En la presente contribución, se trata de demostrar la importancia de este pensamiento para el análisis del desempleo. Las series de datos de la UNCTAD, ahora disponibles, apoyan de una manera dramática esta perspectiva "latinoamericana" del desarrollo Europeo. Prácticamente durante todas las décadas decisivas de los años ochenta, noventa $y$ en la década actual, tanto la penetración de Europa por el capital transnacional como el flujo del capital nacional hacia otros mercados - con sus respectivos efectos negativos y positivos sobre el empleo- han sido mucho mayor en Europa que en los Estados Unidos $^{2}$.

Paradójicamente, es Estados Unidos, el menos "globalizado" en comparación con la mayoría de los países Europeos.

$2 \quad$ Fuente: nuestras propias compilaciones con base en datos coleccionados de la UNCTAD. En: $<$ http://www.unctad.org/sections/dite_dir/ docs/wir2007_instock_gdp_en.xls>. Además, <http://www.unctad.org/Templates/Page. asp?intItemID $=3198 \&$ lang $=1>\quad<$ http:// www.unctad.org/en/docs/wir2008_en.pdf > <http://www.unctad.org/Templates/Page. asp?intItemID=3277\&lang=1> 


\section{GRÁFICO 1 \\ EL DESARROLLO DE LAS INVERSIONES EXTRANJERAS EN LOS ESTADOS UNIDOS Y EN EUROPA (COMO \% DEL PIB)}

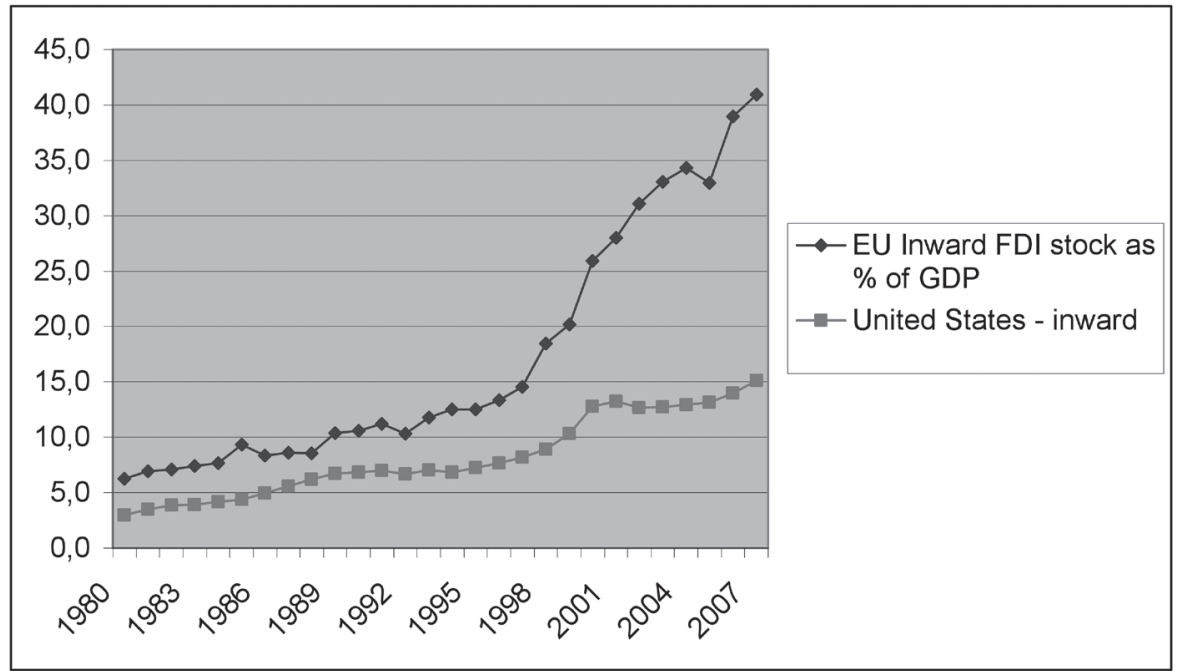

Nota: el cuadro muestra que la penetración por el capital extranjero está mucho más alta en Europa que en Estados Unidos.

Estos desequilibrios también existen tanto en la época de las administraciones republicanas de Reagan, Bush (padre) y Bush (hijo) como durante los 8 años de la administración demócrata bajo Clinton.

El presente artículo pone su énfasis sobre el problema social actual número uno en Europa - el empleo-y trata de demostrar con métodos del avanzado análisis de datos estadísticos "cross-nacionales", de que la dependencia - sobre todo de los consorcios transnacionales- desempeña un papel significativo, no solo como freno del llamado "proceso de Lisboa" para alcanzar los niveles estadounidenses en 2010, sino también del empleo en Europa. Otra vez, este tipo de relaciones lleva a plantear la hipótesis que Europa precisaría volver a la búsqueda de una repuesta en términos de política industrial en la línea preconisada en los años del Comisario de la Unión Europea, Jacques Delors.

Esta tesis provocante, por lo seguro, para muchos, precisa una explicación más profunda. Autores como Joseph Alois Schumpeter y más tarde, economistas y sociólogos como Volker Bornschier, Fernando Henrique Cardoso, Michal Kalecki, Raúl Prebisch, Kurt Rothschild y Osvaldo Sunkel han sido siempre conscientes de las crisis cíclicas y de los desequilibrios regionales, $y$ de los auges y el declive de regiones enteras e incluso continentes en el proceso de desarrollo capitalista. Estas tradiciones de la teoría económica también subrayaron la importancia de los efectos de la competencia monopolística - con sus consecuencias negativas para el crecimiento económico a largo plazo. Ya en la "vieja Galicia" del imperio Austriaco-Húngaro de 1909 a 1911, Joseph Alois Schumpeter obtuvo valiosos conocimientos sobre la naturaleza del desarrollo mundial en la periferia. El entonces joven profesor podía observar de primera mano en el caso de Galicia, la "destrucción creativa", que constituyó el capitalismo en la periferia del Imperio. Varias de sus obras más importantes, como The Nature and Essence of Theoretical Economics (1908), The Theory of Economic Development: An inquiry into profits, capital, credit, interest and the business cycle (1911) fueron muy influenciados por estas primeras experiencias en la periferia del entonces imperio, $y$ no por la experiencia del "centro". Al igual de muchos otros teóricos del desarrollo de la primera generación después de la Segunda Guerra 
Mundial, como Kurt Mandelbaum, Paul Narcyz Rosenstein-Rodan y Hans Wolfgang Singer, Schumpeter compartió la observación de que el capitalismo nunca es un proceso de equilibrio. Mandelbaum, Rosenstein-Rodan y Singer estaban profundamente convencidos de que el capitalismo, además, no significa automáticamente crecimiento sin crisis, pleno empleo, la sostenibilidad del medio ambiente y el fin de la exclusión social. El término "destrucción creativa”, introducido por Schumpeter, describe el proceso de la transformación que acompaña a la innovación radical, realizada por parte de los empresarios innovadores, es la fuerza que sostiene a largo plazo el crecimiento económico, a pesar de ( o merced de) que destruye las rentas de las empresas establecidas que gozan de cierto grado de poder monopolístico. Nosotros vemos mucha evidencia en favor de ese proceso que permite el progreso y la erradicación de las rentas monopolísticas estáticas. Hoy en día, existe en las Ciencias Políticas y en la Sociología una masiva literatura, con más de 300 estudios publicados que son a menudo diametralmente opuestos a la línea optimista de la Comisión Europea de los últimos años que mantiene la necesidad de continuar $y$ profundizar el proceso de la globalización (Fuente: nuestras propias compilaciones con base en ISI Web of Knowledge, un producto de Thomson Reuters, consulta 4 de Julio de 2009. En: <http://www.isiwebofknowledge.com/>).

Por falta de datos comparativos, esta literatura ha hecho, hasta ahora, sólo una breve mención al problema del desempleo (Alderson, 1999; Barbieri, K. y Reuveny, R., 2005; Bornschier, V., 1981; Bornschier y Hoby, 1981; Firebaugh,1992; Gustafsson y Johansson, 1999; Kentor, 1998; Muller, 1985; Paul y Barbato, 1985; Precehel, 2009; Richards, Gelleny y Sacko, 2001; Tausch, 2003, 2004, 2007; Wimberley, 1990). [Fuente: nuestras propias compilaciones con base en los sistemas de texto entero de documentación, EBSCO Host ${ }^{3}$ y JSTOR ${ }^{4}$, consultados 4 de julio de 2009. Acceso

3 http://www.ebscohost.com/

4 http://www.jstor.org/ posible desde muchas bibliotecas universitarias $y$ académicas en el mundo; el acceso del autor a EBSCO Host y JSTOR fue realizado por la biblioteca universitaria de Innsbruck, Austria].

Parece importante a esta altura destacar que en este artículo, el indicador de la dependencia principal es MNC PEN que mide la penetración de un país por el capital internacional, es decir, los porcentajes (documentados por UNCTAD) de las inversiones de las empresas multinacionales como porcentaje del PIB total del país anfitrión.

Los conocidos efectos de aceleración del desarrollo y madurez de desarrollo tienen que ser calificados de manera importante también en este contexto. Desde los días del economista estadounidense, Simon Kuznets, muchos investigadores han aplicado una curva no-lineal para captar estos efectos. La curva de la función no-lineal del desempleo, significa que hay una aceleración del desempleo en los países de renta media. Kuznets ha sido el primero en introducir una "luz al final del túnel" en el debate sobre el desarrollo de la desigualdad: en primer lugar las cosas se ponen peor, $y$ mejoran más tarde. Eso significa que la "gran transformación" abarca tanto efectos negativos como positivos para las economías, pero el proceso del desarrollo acaba produciendo un resultado neto positivo. Sin embargo, las Ciencias Sociales deben poder llegar a clarificar en qué medida este resultado neto positivo se explica por políticas adecuadas o difiere de un país al otro según precisamente el tipo de políticas o de intervención de los poderes públicos. De tal manera se podría avanzar en la definición de una respuesta europea voluntarista para sacar mejor provecho de la globalización.

A partir de los escritos de Perroux, Prebisch y Rothschild en la década de 1930, creemos que a pesar de la cantidad de empleo, 'el empleo a largo plazo' de la semi-periferia será desequilibrado. El capitalismo en la semi-periferia, como en el centro, se caracteriza por fuertes fluctuaciones cíclicas. El surgimiento de un grupo de semi-periferias tendería así a lograrse a costa de otro grupo, en la medida que la diversa estructura de la economía mundial, basada en la desigualdad de la transferencia, tendería a permanecer estable. En 1979, Fernando 
Henrique Cardoso resumía la esencia de las teorías de la dependencia de la siguiente manera:

$\diamond \quad$ Hay una penetración financiera y tecnológica por los países desarrollados de los centros capitalistas en los países de la periferia y la semi-periferia.

$\diamond \quad$ Esto produce un desequilibrio en la estructura económica tanto dentro de las sociedades de la periferia $y$ entre estas $y$ los centros.

$\diamond \quad$ Lo que frena el crecimiento sostenido en la (semi-) periferia.

$\diamond \quad$ Favorece modelos específicos de las relaciones de clase.

$\diamond \quad$ Esto exige modificaciones en el papel del Estado para garantizar el funciona- miento de la economía y exige la articulación política de una sociedad, que contiene, en sí misma, focos de desequilibrio estructural (Cardoso, 1979).

Un aumento del grado de la monopolización en el centro determina, con el fin de mantener la cuota de los salarios al menos constante, un aumento de la explotación de los productores de materia prima en la semi-periferia y periferia para compensar el equilibrio.

Treinta años después, convendría saber en qué medida la aceleración del desarrollo mundial por las economías emergentes ha permitido reducir o no el grado de monopolización del centro, tomando en cuenta el fenómeno de la penetración por las transnacionales.

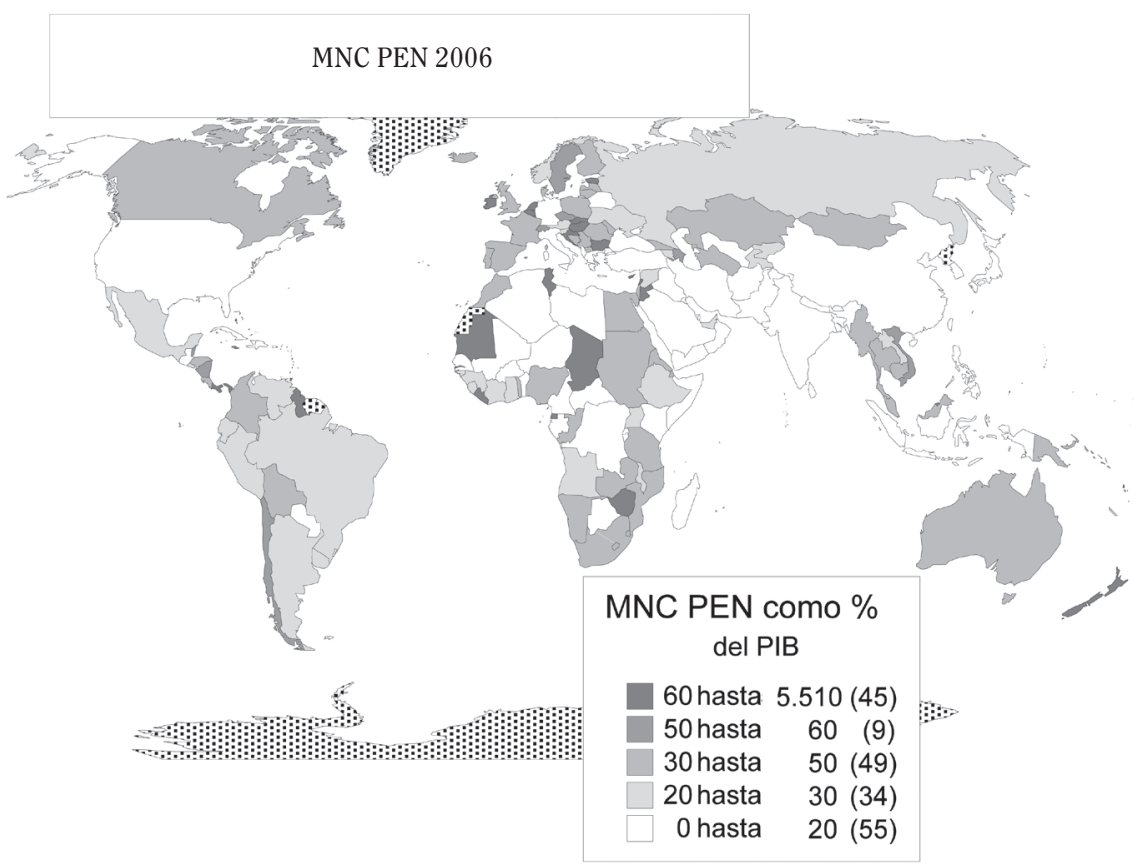

Fuente: nuestras propias compilaciones con base en: datos coleccionados de la UNCTAD http://www.unctad.org/sections/ dite_dir/docs/wir2007_instock_gdp_en.xls. Además, http://www.unctad.org/Templates/Page.asp?intItemID=3198\&lang=1 $y$ http://www.unctad.org/en/docs/wir2008_en.pdf; $y$ http://www.unctad.org/Templates/Page.asp?intItemID=3277

\&lang $=1$

Color oscuro: alta penetración por el capital extranjero. Europa, después de seis años de competición con los Estados Unidos, es uno de los continentes más penetrados por el capital internacional en todo el mundo. 
LOS RESULTADOS DE NUESTRA INVESTIGACIÓN AL NIVEL DE 127 PAÍSES DEL MUNDO CON DATOS COMPLETOS

Nuestro "modelo" no pretende llegar a una explicación del desempleo ni del funcionamiento del proceso de desarrollo, sino poner en evidencia ciertas correlaciones susceptibles de detectar relaciones estructurales insuficientemente consideradas en los "modelos" explicativos de la profesión. En términos de la ecuación verificada con los datos empíricos, la variable dependiente es la siguiente:

$\diamond \quad$ desempleo (Naciones Unidas, datos más recientes, alrededor de 2007).

Los parámetros económicos y sociológicos usados como "variables independientes" en nuestra ecuación comprenden la siguiente lista (véase también Durlauf, et ál.
2008; Tausch, 2003, 2004, 2007). La documentación de las variables se encuentra en el apéndice:

$\diamond \quad \%$ población mundial (población del país como \% de la población mundial)

$\diamond \quad$ DYN MNC PEN-incremento de la penetración por el capital extranjero

$\diamond \quad$ gasto público en educación por PNB

$\diamond \quad$ logaritmo natural del ingreso per cápita $\wedge 2$

$\diamond \quad$ logaritmo natural del PIB per cápita

$\diamond \quad$ los gastos de salud por producto bruto interno (PIB)

$\diamond \quad$ MNC PEN 1995 la penetración por el capital transnacional

$\diamond \quad$ tasa anual de crecimiento demográfico, 1975-2005 (\%).

La relación entre nivel de desarrollo y la variable dependiente es la siguiente:

DESEMPLEO Y NIVEL DEL DESARROLLO ${ }^{5}$

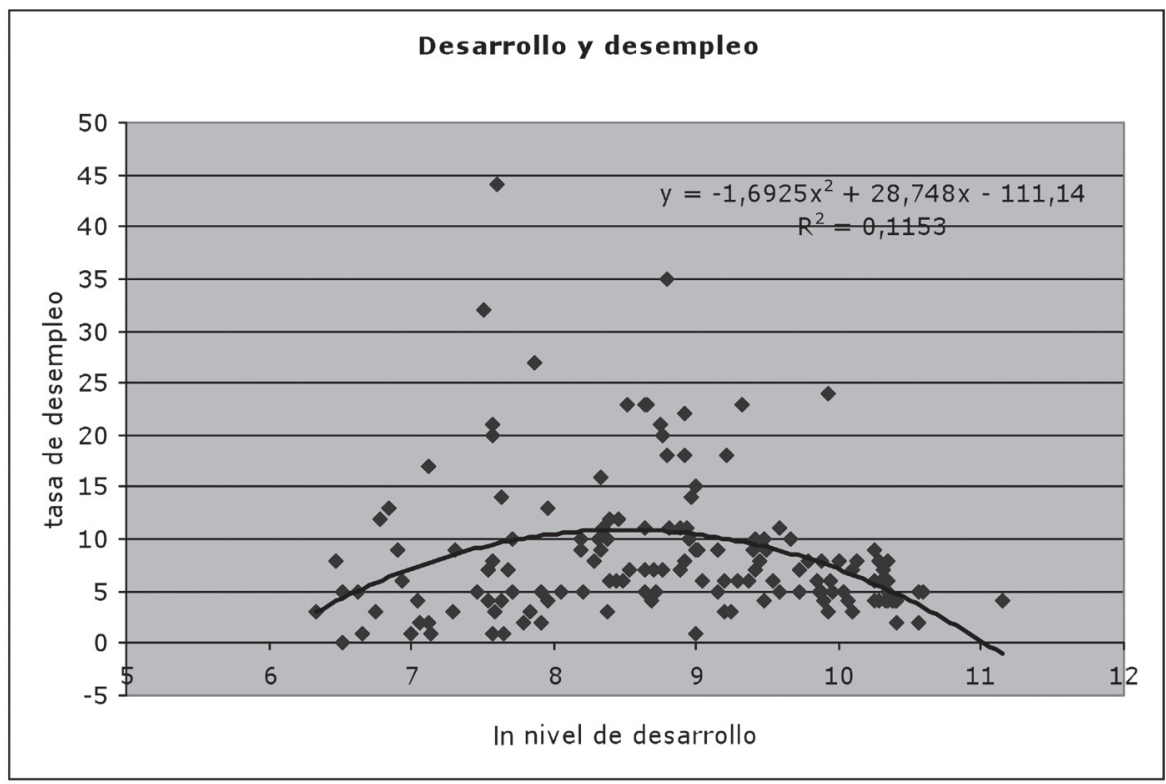

Nota: el cuadro muestra que el desempleo en el sistema mundial está más alto a medianos niveles del desarrollo.

$5=$ al +- bl* primera parte curvilínea función del nivel de desarrollo +-b2* segunda parte curvilínea función del nivel de desarrollo, etc. 
Independiente de esta función, los resultados presentados en el cuadro muestran que la penetración del capital extranjero tiene un efecto estructural muy negativo a largo plazo sobre el empleo. Esta correlación -incluso antes de poder identificar sus mecanismos subyacentes - lleva a cuestionar en qué medida la crisis del empleo Europeo es sobre todo una crisis de la falta de la política industrial en nuestro continente $y$ de la estrategia ultraliberal de la Comisión Europea durante los últimos años.

TABLA 1

DETERMINANTES DEL DESEMPLEO

COEFICIENTES (a)

\begin{tabular}{|c|c|c|c|c|c|}
\hline & $\mathrm{B}$ & $\begin{array}{c}\text { ERROR } \\
\text { ESTANDARIZADO }\end{array}$ & BETA & $T$ & $\begin{array}{c}\text { ERROR } \\
\text { ESTANDARIZADOP }\end{array}$ \\
\hline (Constante) & $-103,446$ & 26,865 & & $-3,851$ &, 000 \\
\hline \% De la población mundial &,- 231 & ,191 &,- 095 & $-1,210$ & ,229 \\
\hline $\begin{array}{l}\text { Promedio anual del crecimiento de la población, } \\
\text { 1975-2005 (\%) }\end{array}$ &,- 326 & ,464 &,- 064 &,- 703 & ,484 \\
\hline MNC PEN 1995 & 075 & ,019 & ,319 & 3,862 & ,000 \\
\hline DYN MNC PEN 1995/2000 &,- 022 & ,019 &,- 098 & $-1,161$ & ,248 \\
\hline Gasto publico en educación como \% del PNB & ,659 & ,264 & ,212 & 2,494 & ,014 \\
\hline Ln PIB per capita & 26,243 & 6,168 & 4,926 & 4,255 &, 000 \\
\hline Ln PIB per capita $* * 2$ & $-1,561$ & ,351 & $-5,123$ & $-4,441$ &, 000 \\
\hline
\end{tabular}

a ) Variable dependiente: VAR00085, $\mathrm{R}^{\wedge} 2$ corregido $=26.2 \%$, df $=126 ; \mathrm{F}=7.385, \mathrm{p}=.000$, (pin <.10; pout $\left.<.15\right)$. El coeficiente de beta más alto que 1.0 se debe a la co-linealidad de la función del cuadro 3. Calculaciones a base de SPSS, XV.

\section{HACIA UNA PERSPECTIVA REGIONAL}

Con base en los datos de Dirección General de Política Regional de la Comisión Europea, sobre el desarrollo regional en Europa tratamos de establecer si esta hipótesis de la influencia negativa de la penetración multinacional sobre el empleo se puede confirmar al nivel europeo de más de 300 regiones, que forman parte de la base de datos libremente accesible de la Comisión Europea sobre el tema <http://ec.europa.eu/regional_policy/sources/docoffic/official/reports/cohesion4/index_ en.htm>. Nuestras variables son tomadas de la base de datos de tipo Microsoft Excel de la Comisión, así que nuestra presentación va a ser muy cuantitativa. Los datos se refieren a mediados de la década actual. Los parámetros tomados como variables independientes en la ecuación son: $\diamond \quad$ estructura de edad de la población: $\%<15$

$\diamond \quad$ estructura de edad de la población: \% 15 $-64$

$\diamond \quad \%$ empleo en la agricultura

$\diamond \quad \%$ de la población adulta con educación alta

$\diamond \quad \%$ de la población adulta con educación mediana

$\diamond \quad$ FDI: Intensidad del empleo al nivel regional en sucursales de las transnacionales (escala de 5 grados, basada en el empleo en sucursales de las transnacionales como \% del empleo regional total, tomada de la publicación de la Comisión Europea, <http:// ec.europa.eu/regional_policy/sources/ docoffic/official/reports/cohesion4/ pdf/4cr_en.pdf>

$\diamond \quad$ PIB per capita regional (poder adquisitivo) (Índice, EU 27=100), 2004 
$\diamond \quad$ PIB per capita regional (Índice, EU $27=100), 2004^{\wedge} 2$

$\diamond \quad \%$ empleo en la industria

$\diamond \quad$ Densidad de la población (hab./ $\mathrm{Km}^{2}$ ), 2004

$\diamond \quad$ Crecimiento de la población, 1995-2004

$\diamond \quad$ Gasto de investigación y desarrollo (I\&D) como \% del PIB, 2004

$\diamond \quad$ Población total 2004

Con estas variables tratamos de medir el grado de correlación con las siguentes variables dependientes:

$\diamond \quad$ tasa de crecimiento regional
$\diamond \quad$ tasa de desempleo regional de la gente
con edad

$\diamond \quad$ tasa de desempleo regional

$\diamond \quad$ Indice de Lisboa regional

Para la interpretación final, simplemente hemos utilizado la expresión verbal positiva de los fenómenos, con los cambios correspondientes en los signos matemáticos (teniendo en cuenta, que el desempleo es un fenómeno negativo). Independientemente del nivel regional de desarrollo, queda claro que la intensidad del empleo al nivel regional en sucursales de las transnacionales tiene efectos muy negativos sobre el crecimiento regional, el empleo, el empleo de la gente con edad, y el indice regional del proceso de Lisboa, calculado por la Comisión Europea:

TABLA 2

EL FRACASO DEL DESARROLLO REGIONAL EN EUROPA Y LA PENETRACIÓN MULTINACIONAL

\begin{tabular}{|c|c|c|c|c|c|c|c|c|}
\hline $\begin{array}{l}\text { Indicador } \\
\text { región al }\end{array}$ & $\begin{array}{l}\text { Beta } \\
\text { creci- } \\
\text { miento }\end{array}$ & $\begin{array}{l}\text { error } p \text { : } \\
\text { creci- } \\
\text { miento }\end{array}$ & $\begin{array}{l}\text { Beta empleo } \\
\text { de la gente } \\
\text { con edad }\end{array}$ & $\begin{array}{l}\text { error } p \text { : } \\
\text { empleo de } \\
\text { la gente } \\
\text { con edad }\end{array}$ & $\begin{array}{l}\text { Beta } \\
\text { evitando } \\
\text { desempleo }\end{array}$ & $\begin{array}{l}\text { error } p \text { : } \\
\text { evitando } \\
\text { desempleo }\end{array}$ & $\begin{array}{l}\text { Beta } \\
\text { Índice de } \\
\text { Lisboa } \\
\text { regional }\end{array}$ & $\begin{array}{l}\text { error } p \text { : } \\
\text { Índice de } \\
\text { Lisboa } \\
\text { regional }\end{array}$ \\
\hline $\begin{array}{l}\text { Estructura } \\
\text { de edad de la } \\
\text { población: } \% \\
<15\end{array}$ & 0,128 & 0,045 & 0,096 & 0,150 & 0,119 & 0,044 & 0,097 & 0,003 \\
\hline $\begin{array}{l}\text { Estructura } \\
\text { de edad de la } \\
\text { población: } \% \\
15-64\end{array}$ & 0,240 & 0,001 & $-0,154$ & 0,038 & $-0,089$ & 0,173 & $-0,155$ & 0,000 \\
\hline $\begin{array}{l}\% \text { empleo en la } \\
\text { agricultura }\end{array}$ & 0,005 & 0,947 & 0,299 & 0,001 & 0,052 & 0,488 & 0,226 & 0,000 \\
\hline $\begin{array}{l}\text { \% de la población } \\
\text { adulta con } \\
\text { educación alta }\end{array}$ & 0,443 & 0,000 & 0,441 & 0,000 & 0,169 & 0,011 & 0,302 & 0,000 \\
\hline $\begin{array}{l}\% \text { de la población } \\
\text { adulta con } \\
\text { educación } \\
\text { mediana }\end{array}$ & $-0,079$ & 0,262 & $-0,001$ & 0,989 & $-0,135$ & 0,038 & 0,182 & 0,000 \\
\hline $\begin{array}{l}\text { FDI: Intensidad } \\
\text { del empleo al } \\
\text { nivel regional en } \\
\text { sucursales de las } \\
\text { transnacionales } \\
\text { (escala, basada } \\
\text { en tal empleo } \\
\text { como \% del } \\
\text { empleo regional } \\
\text { total) }\end{array}$ & $-0,383$ & 0,000 & $-0,148$ & 0,052 & $-0,294$ & 0,000 & $-0,207$ & 0,000 \\
\hline
\end{tabular}


TABLA 2 (CONTINUACIÓN)

EL FRACASO DEL DESARROLLO REGIONAL EN EUROPA Y LA PENETRACIÓN MULTINACIONAL

\begin{tabular}{|c|c|c|c|c|c|c|c|c|}
\hline $\begin{array}{l}\text { Indicador } \\
\text { región al }\end{array}$ & $\begin{array}{l}\text { Beta } \\
\text { creci- } \\
\text { miento }\end{array}$ & $\begin{array}{l}\text { error } p \text { : } \\
\text { creci- } \\
\text { miento }\end{array}$ & $\begin{array}{l}\text { Beta empleo } \\
\text { de la gente } \\
\text { con edad }\end{array}$ & $\begin{array}{l}\text { error } p \text { : } \\
\text { empleo de } \\
\text { la gente } \\
\text { con edad }\end{array}$ & $\begin{array}{l}\text { Beta } \\
\text { evitando } \\
\text { desempleo }\end{array}$ & $\begin{array}{l}\text { error } p \text { : } \\
\text { evitando } \\
\text { desempleo }\end{array}$ & $\begin{array}{l}\text { Beta } \\
\text { Índice de } \\
\text { Lisboa } \\
\text { regional }\end{array}$ & $\begin{array}{l}\text { error } p \text { : } \\
\text { Índice de } \\
\text { Lisboa } \\
\text { regional }\end{array}$ \\
\hline $\begin{array}{l}\text { PIB per capita } \\
\text { regional (poder } \\
\text { adquisitivo) } \\
\text { (Indice, EU } \\
27=100 \text { ), } 2004\end{array}$ & $-0,537$ & 0,051 & 1,094 & 0,000 & 1,300 & 0,000 & 1,921 & 0,000 \\
\hline $\begin{array}{l}\text { PIB per capita } \\
\text { regional (Indice, } \\
\text { EU } 27=100 \text { ), } \\
2004^{\wedge} 2\end{array}$ & 0,449 & 0,060 & $-0,846$ & 0,001 & $-0,724$ & 0,001 & $-1,174$ & 0,000 \\
\hline $\begin{array}{l}\% \text { empleo en la } \\
\text { industria }\end{array}$ & 0,026 & 0,703 & 0,020 & 0,779 & 0,126 & 0,045 & 0,127 & 0,000 \\
\hline $\begin{array}{l}\text { Densidad de la } \\
\text { población (hab./ } \\
\mathrm{Km}^{2} \text { ), } 2004\end{array}$ & $-0,224$ & 0,002 & $-0,031$ & 0,669 & $-0,229$ & 0,000 & $-0,103$ & 0,004 \\
\hline $\begin{array}{l}\text { Crecimiento de la } \\
\text { población, 1995- } \\
2004\end{array}$ & 0,281 & 0,000 & 0,018 & 0,828 & 0,171 & 0,018 & 0,083 & 0,037 \\
\hline $\begin{array}{l}\text { Gasto } \\
\text { investigación } \\
\text { (R\&D) como \% } \\
\text { del PIB, } 2004\end{array}$ & $-0,083$ & 0,234 & 0,137 & 0,062 & 0,000 & 0,997 & 0,179 & 0,000 \\
\hline $\begin{array}{l}\text { Población total } \\
2004\end{array}$ & 0,028 & 0,617 & $-0,137$ & 0,021 & $-0,096$ & 0,066 & $-0,060$ & 0,037 \\
\hline
\end{tabular}

Fuente: nuestras propias calculaciones a base de <http://ec.europa.eu/regional_policy/sources/docoffic/official/reports/ cohesion $4 /$ pdf/4cr_en.pdf $>$ Calculaciones a base de SPSS, XV.

\section{CALIDAD ESTADÍSTICA DE LOS MODELOS}

\begin{tabular}{lllll}
\hline & error $\mathrm{p}$ & $\mathrm{R}^{\wedge} 2$ & $\mathrm{n}=$ & $\mathrm{F}=$ \\
\hline crecimiento regional & .000 & 42,10 & 218 & 13,16 \\
tasa de desempleo de la gente con edad & .000 & 36,40 & 218 & 10,55 \\
tasa de desempleo & .000 & 50,60 & 218 & 18,08 \\
implementación del proceso de Lisboa & .000 & 85,10 & 218 & 95,97 \\
\hline
\end{tabular}

\section{DISCUSIÓN DE LOS RESULTADOS}

Hace casi cuatro décadas, el economista Chileno Osvaldo Sunkel dijo:

The advancement of modernization introduces, so to speak, a wedge along the area dividing the integrated from the segregated segments (...) In this process, some national entrepreneurs are incorporated as executives into the new enterprises or those absorbed by the TRANCO (i.e. transnational corporations), and others are marginalized; some professionals, forming part of the technical staff and the segment of employees are incorporated, and the rest are marginalized; part of the qualified labor supply and 
those that are considered fit to be upgraded are incorporated, while the remainder are marginalized ${ }^{6}$ (1973: 18-42).

Hoy en día, esta prognosis se cumple con fuerza, $y$ cabe plantearse en qué medida sería la razón más profunda de la crisis en la cual Europa se encuentra actualmente. Para destacar todavía más esta perspectiva, comparamos aquí — por vez primera en la literatura - el suceso de la política de empleo al nivel regional en todos los países del OCDE. Solo unas islas del bienestar lograron en Europa un desempleo debajo de $6 \%$ [antes del reciente tsunami financiero], mientras que en Japón y Australia, por ejemplo, altas tasas de desempleo fueron casi totalmente desconocidas, $y$ en los EEUU solo los estados de Alaska, Oregón, Luisiana, Alabama, Kentucky y Carolina del Sur mostraron tales tasas altas de desempleo. En aproximadamente $2 / 3$ del territorio de toda la Unión Europea, tales tasas de desempleo son típicas. En Canadá, la crisis del empleo es más común en el norte del país, en Ontario y en las provincias del Atlántico. Fuente: nuestras propios

$6 \quad$ "Introduce el adelanto de la modernización, por así decirlo, una cuña a lo largo de la zona dividiendo el integrado de los segmentos segregados (...) En este proceso, algunos empresarios nacionales se incorporan como directivos en las nuevas empresas o aquellas absorbidas por el TRANCO (es decir, transnacionales), y otros están marginados; se incorporan algunos profesionales, formando parte del equipo técnico y el segmento de los empleados, y el resto están marginados; parte de la oferta de mano de obra calificada y de los que se consideran aptos para actualizarse se incorporan, mientras que el resto está marginado". cálculos con base en <http://stats.oecd.org/ oECDregionalstatistics/indexTL2.html>.

Nuestra discusión debe mencionar también el punto clave de la relación de las inversiones del capital transnacional con el seguro social. Agradecemos este punto a un "referee" anónimo de esta Revista quién, en su opinión, hizo hincapié en el hecho de que

... diría que el problema mayor es que la tasa de desempleo refleja cosas distintas en varios países. Si bien está más elevada en la Unión Europea, se justifica en parte por la amplitud de la cobertura social, mientras en otras regiones del mundo se encuentra más gente afuera de las estadísticas del desempleo porque no tienen derecho a un programa de ayuda a los desempleados, porque trabajan en la economía informal, porque son trabajadores sub-empleados o porque ya expiró su periodo como beneficiarios del apoyo del gobierno.

Nuestro análisis demuestra precisamente la validez de este punto; $y$ de que la fuerza del capital transnacional lamentablemente tiende a debilitar los mecanismos del seguro social, que protegen todavía - sobre todo en Europa - a la gente de las tendencias masivas de la desigualdad. Asimismo, este demuestra la determinación del gasto del seguro social como $\%$ del PIB (según los datos del OIT: social security expenditure per GDP average 1990s (ILO), ILO <http://www-ilo-mirror.cornell.edu/public/ english/protection/socfas/research/stat/table14. htm>: 
TABLA 3

LA DETERMINACIÓN DE LOS GASTOS DEL SEGURO SOCIAL (COMO PORCENTAJE DEL PIB, SEGÚN DATOS DEL OIT) A ESCALA MUNDIAL

\begin{tabular}{lccccc}
\hline VARIABLE INDEPENDIENTE & $\mathrm{B}$ & $\begin{array}{c}\text { ERROR } \\
\text { STANDARIZADO }\end{array}$ & $\begin{array}{c}\text { BETA } \\
\text { VALOR DE } \\
\text { T- }\end{array}$ & $\begin{array}{c}\text { PROBABILIDAD } \\
\text { DEL ERROR P }\end{array}$ \\
\hline (Constante) & 130,138 & 23,357 & & 5,572 & 0,000 \\
\% de la población mundial & $-0,133$ & 0,156 & $-0,042$ & $-0,852$ & 0,396 \\
Promedio anual del crecimiento de la & $-3,737$ & 0,553 & $-0,442$ & $-6,752$ & 0,000 \\
población, 1975-2005 (\%) & & & & & \\
LN PIB per capita & $-30,587$ & 5,307 & $-3,961$ & $-5,763$ & 0,000 \\
LN PIB per capita ^2 & 1,915 & 0,300 & 4,340 & 6,387 & 0,000 \\
MNC PEN - 1995 & $-0,071$ & 0,024 & $-0,146$ & $-2,937$ & 0,004 \\
MNC PEN: DYN MNC PEN 1995-2005 & 0,017 & 0,019 & 0,045 & 0,881 & 0,381 \\
Gasto público en educación como \% del & 1,060 & 0,260 & 0,219 & 4,072 & 0,000 \\
PIB & & & & & \\
Calidad estadística de los modelos & adj. R^2 & $\mathrm{n}$ & $\mathrm{F}$ & probabilidad \\
& & & & del error & \\
\hline & 78,500 & 98 & 52,056 & 0.000 & \\
\hline
\end{tabular}

Fuente: Propios cálculos con base en nuestros datos, libremente accesibles en la red bajo la dirección: http://www. hichemkaroui.com/?p=1317; cálculo con base en el paquete estadístico de SPSS, XV.

La penetración por el capital transnacional es correlacionada de manera significativa con una reducción de la protección social. Los europeos, al admitir estas estructuras, pronto o más tarde pagarán la cuenta. Se dice muchas veces en la prensa internacional de que la presidencia de Barack Obama significa un cambio en los modelos neo-liberales que antes existían en Estados Unidos. El análisis empírico demuestra una tendencia completamente distinta y todavía más seria en sus implicaciones. No hay, hasta ahora, un esfuerzo pan-europeo para efectuar tal cambio en el modelo político $y$ económico. En verdad, y pese a toda la retórica del «modelo social Europeo», son precisamente los europeos quienes practicaron durante las últimas décadas una apertura de los mercados sin precedente, $y$ quienes nunca alcanzaron los niveles de la política industrial silenciosa de las administraciones republicanas de los últimos años en los Estados Unidos. Puede ser, que nuestro análisis sea una provocación, pero los datos empíricos no permiten dejar de lado esta hipótesis provocante: son los Estados Unidos, que se benefician del hecho, que son mucho menos globalizados que los europeos. Y en vez de reaccionar a tal situación con una política razonable de la estructura industrial, Europa empuja cada vez más su camino hacia la apertura de los mercados y estructuras económicas sin las herramientas adecuadas. Así, Europa debería aprender algo de la experiencia de América Latina.

\section{FUENTES DE DATOS ESTADÍSTICOS PARA LA COMPARACIÓN MACRO-CUANTITATIVA}

\section{FUENTES}

1. UNCTAD <http://www.unctad.org/ sections/dite_dir/docs/wir2007_instock_ gdp_en.xls>

2. United Nations Statistics <http:// unstats.un.org/unsd/Demographic/ Products/socind/unemployment.htm> 
3. UNDP Human Development Report Office $<$ http: //hdr.undp.org/en/statistics/data/>

4. United States Central Intelligence Agency <http: //www.photius. com/rankings/spreadsheets_2008/ population_2008.xls>

$\diamond \quad$ MNC PEN increase in MNC penetration 1995-2000

$\diamond \quad$ MNC PEN 1995.

Datos coleccionados UNDP Human Development Report Office <http://hdr.undp. org/en/statistics/data/>

$\begin{array}{ll}\diamond & \% \text { world population } \\ \diamond & \text { Annual population growth rate, 1975- } \\ & 2005(\%) \\ \diamond & \text { DYN } 1990-2005 \text { real economic growth } \\ & \text { rate } \\ \diamond & \text { ln GDP per capita in PPP } \$ \\ \diamond & \text { ln GDP per capita in PPP } \$ \wedge 2 \\ \diamond & \text { total population, } 2005 .\end{array}$

Datos coleccionados United Nations Statistics <http://unstats.un.org/unsd/ Demographic/Products/socind/unemployment. htm>

$\diamond \quad$ Unemployment rate, latest available year.

Datos coleccionados United States Central Intelligence Agency <http://www. photius.com/rankings/spreadsheets_2008/ population_2008.xls $>$ (based on US CIA)

$\diamond \quad \%$ world population

$\diamond \quad$ Annual population growth rate, 19752005 (\%).

\section{LITERATURA}

Alderson, A. S. "Income Inequality, Development, and Dependence: A Reconsideration". American Sociological Review 64 (4). Aug., 1999: 606-631.

Barbieri, K. and Reuveny, R. "Economic Globalization and Civil War". Journal of Politics 67 Issue 4. Nov. 2005:1228-1247.
Barro, R. J. and Sala-i-Martin, X. Economic Growth. 2nd ed. Cambridge. MA: MIT Press, 2004.

Beer, L. "Income Inequality and Transnational Corporate Penetration". Journal of World Systems Research 5 (1). 1999:1-25.

Bornschier, V. "Income Inequality. A CrossNational Study of the Relationships between MNC Penetration, Dimensions of the Power Structure and Income Distribution". Together with ThanhHuyen Ballmer-Cao. American Sociological Review 44 (3). 1979: 487506.

Bornschier, V. "Dependent Industrialization in the World Economy: Some Comments and Results concerning a Recent Debate". The Journal of Conflict Resolution 25 (3) Sep., 1981: 371-400.

Bornschier, V. and Ballmer-Cao, T. H. "Income Inequality: A Cross-National Study of the Relationships Between MNC-Penetration, Dimensions of the Power Structure and Income Distribution". American Sociological Review 44. 1979:438-506.

Bornschier, V. and Chase-Dunn, Ch. K. Transnational Corporations and Underdevelopment. NY, NY: Praeger, 1985.

Bornschier, V. and Hoby, J. P. "Economic Policy and Multinational Corporations in Development: The Measurable Impacts in Cross-National Perspective". Social Problems 28 (4). Development Processes and Problems. Apr., 1981: 363-377.

Bornschier, V.; Chase-Dunn, Christopher and Rubinson, Richard. "Cross-National Evidence of the Effects of Foreign Investment and Aid on Economic Growth and Inequality: A Survey of Findings and a Reanalysis". American Journal of Sociology 84 (3). 1978: 651-683. 
Cardoso, F. H. and Faletto, E. Dependencia y desarrollo en América Latina. México DF: Editorial siglo XXI, 1971.

Chase-Dunn, Ch. K. "The Effects of International Economic Dependence on Development and Inequality: a Crossnational Study". American Sociological Review 40. 1975: 720-738.

Durlauf, St. N.; Kourtellos A., Andros and Tan Ch., M. "Are any Growth Theories Robust?". The Economic Journal 118. March 2008: 329-346.

Esposito, P. and Steher, R. "Effects of HighTech Capital, FDI and Outsourcing on Demand for Skills in West and East". Working Papers 51. Vienna Institute for International Economic Studies, 2009.

Firebaugh, G. "Growth Effects of Foreign and Domestic Investment". The American Journal of Sociology 98 (1). Jul., 1992: 105-130.

Frank, A. G. ReOrient: Global Economy in the Asian Age. Ewing, USA: University of California Press, 1998.

Galtung, J. "A Structural Theory of Imperialism". Journal of Peace Research 8 (2). 1971: 81-117.

Gustafsson, B. and Johansson, M. "In Search of Smoking Guns: What Makes Income Inequality Vary over Time in Different Countries?". American Sociological Review 64 (4). Aug., 1999: 585-605.

Kalecki, M. The Last Phase in the Transformation of Capitalism. New York: Monthly Review Press, 1972.

Kalecki, M. Essays on Developing Economies. With an Introduction by Professor Joan Robinson. Hassocks, Sussex: The Harvester Press, 1979.
Kentor, J. "The Long-Term Effects of Foreign Investment Dependence on Economic Growth, 1940-1990". The American Journal of Sociology 103 (4). Jan., 1998: 1024-1046.

Kuznets, S. "Economic Growth and Income Inequality". The American Economic Review 45 (1). 1955: 1-28.

Kuznets, S. Modern Economic Growth: Rate, Structure and Spread. New Haven, Ст: Yale U. Press, 1976.

Mandelbaum, K. The industrialisation of backward areas. By K. Mandelbaum, assisted by J. R. L. Schneider. Oxford: B. Blackwell, 1945.

Muller, E. N. "Dependent Economic Development, Aid Dependence on the United States, and Democratic Breakdown in the Third World". International Studies Quarterly 29 (4). Dec., 1985: 445-469.

Paul, K. and Barbato, R. "The Multinational Corporation in the Less Developed Country: The Economic Development Model Versus the North-South Model". Academy of Management Review 10. Issue 1. Jan. 1985: 8-14.

Prebisch, R. "Five Stages in My Thinking on Development". Pioneers in Development. A World Bank Publication. Meier G.M. and Seers D. (Eds.). New York and Oxford. Oxford University Press, 1984: 175-191.

Precehel, H. (Summer 1985). "The Effects of Export, Public Debt, and Development on Income Inequality". The Sociological Quarterly 26 (2). 2009: 213-234.

Richards, D. L.; Gelleny, R. R. and Sacko, D. H. "Money with a Mean Streak? Foreign Economic Penetration and Government 
Respect for Human Rights in Developing Countries". International Studies Quarterly 45 (2). Jun., 2001: 219-239.

Rosenstein-Rodan, P. N. Capital formation and economic development. London: Allen \& Unwin, 1964.

Rothschild, K. W. Marktform, Loehne, Aussenhandel. Vienna: Europa-Verlag, 1966.

Rothschild, K. W. Employment, wages, and income distribution: critical essays in economics. London; New York: Routledge, 1993.

Rubinson, R. "The World Economy and the Distribution of Income within States - a Cross - national Study". American Sociological Review 41 (4). 1976: 638659.

Schumpeter, J. A. The theory of economic development. An inquiry into profits, capital, credit, interest, and the business cycle. Cambridge, Mass: Harvard University Press, 1934.

Schumpeter, J. A. Business cycles. A theoretical, historical, and statistical analysis of the capitalist process. New York, London: McGraw-Hill Book Company inc., 1939.

Schumpeter, J. A. The Process of Creative Destruction. London: Unwin, 1942.

Schumpeter, J. A. (1950). Capitalism, Socialism and Democracy. Third Edition. New York \& London: Harper \& Row, 1975.

Schumpeter, J. A. History of economic analysis. New York: Oxford University Press, 1954.

Schumpeter, J. A. The Theory of Economic Development: An Inquiry into Profits,
Capital, Credit, Interest, and the Business Cycle. Translated by Redvers Opie. London and Oxford: Oxford University Press, 1969.

Schumpeter, J. A. Business cycles: a theoretical, historical, and statistical analysis of the capitalist process. Philadelphia: Porcupine Press, 1982.

Singer, H. W. International development: growth and change. New York, McGrawHill, 1964.

Singer, H. W. and Raffer, K. The economic North-South divide six decades of unequal development. Cheltenham, UK; Northampton, MA, USA: Edward Elgar, 2001.

Sunkel, 0. "Transnational capitalism and national disintegration in Latin America". Social and Economic Studies 22 (1). March 1973: 132-76.

Tausch, A. "Armas socialistas, subdesarrollo $y$ violencia estructural en el Tercer Mundo". Revista Internacional de Sociología 47 (4). CSIC. Madrid, 1989: 583-716.

Tausch, A. (under collaboration of Fred Prager). Towards a Socio-Liberal Theory of World Development. Basingstoke and New York: Macmillan/St. Martin's Press, 1993.

Tausch, A. "Social Cohesion, Sustainable Development and Turkey's Accession to the European Union: Implications from a Global Model". Alternatives: Turkish Journal of International Relations 2. Issue 1. Spring 2003: 1-41.

Tausch, A. "Europe, the Muslim Mediterranean and the End of the Era of Global Confrontation". Alternatives: Turkish Journal of International Relations 3. Issue 4. 2004: 1-29. 
Tausch, A. "Quantitative World System Studies Contradict Current Islamophobia: World Political Cycles, Global Terrorism, and World Development". Alternatives: Turkish Journal of International Relations 6. Issue 1/2. Spring 2007: 15-81.

Tausch, A. (with Christian Gyhmers). From the "Washington" towards a "Vienna Consensus"? A quantitative analysis on globalization, development and global governance. Hauppauge, NY: Nova Science Publishers, 2007.

Tausch, A. and Galindo Lucas, A. "La Unión Europea, la "Ciudad en la Colina" y el proceso de Lisboa". Revista de Trabajo $e$ Inmigración, Ministerio de Trabajo y de Inmigración 77 (1). Madrid, 2009: 69-101.

United Nations (current issues). United Nations Human Development Report. New York and Oxford: Oxford University Press.

United Nations Conference on Trade and Development (current issues). World Investment Report. New York and Geneva: United Nations.
United Nations Department of Economic and Social Affairs (current issues). World Economic and Social Survey. New York: United Nations.

United Nations Economic Commission for Europe (current issues). Economic Survey of Europe. New York: United Nations.

United Nations Economic Commission for Latin America, ECLAC/CEPAL. Globalización y desarrollo. 2002. En: $<$ http://www.eclac.cl/cgi-bin/getProd. asp? $\mathrm{xml}=/$ publicaciones/xml/6/10026/ $\mathrm{P} 10026 . \mathrm{xml} \& \mathrm{xsl}=/ \mathrm{tpl} / \mathrm{p} 9 \mathrm{f} . \mathrm{xsl} \&$ base $=/$ MDG/tpl/top-bottom.xsl>

Wallerstein, I. The Essential Wallerstein. New York: The New Press, 2000.

Wimberley, D. W. "Investment Dependence and Alternative Explanations of Third World Mortality: A Cross-National Study". American Sociological Review 55 (1). Feb., 1990: 75-91. 